\title{
ASO Editorial: Ultrasound as an Extension of our Clinical Examination
}

\author{
Douglas Tyler, $\mathrm{MD}^{1,2}$ \\ ${ }^{1}$ Department of Surgery, University of Texas Medical Branch, Galveston, TX; ${ }^{2}$ Department of Surgical Oncology, \\ University of Texas MD Anderson Cancer Center, Galveston, TX
}

There are many who believe that ultrasound will evolve in the future to be for clinicians what the stethoscope has been in the past. Indeed, improvements in technology and resolution have rapidly expanded the use of ultrasound, allowing it to leave the halls of radiology suites and move into the clinics, emergency rooms, and operating rooms, as well as to the patient's bedside. As ultrasound has expanded, non-radiologist clinicians, and especially surgeons, have learned to embrace the technology to explore a wide variety of clinical scenarios, ranging from vascular examinations, arterial and central line placement, focused assessment with sonography for trauma (FAST) examinations, surveilling nodal basins, and focused evaluations of organs such as the breast and thyroid.

In this issue of Annals of Surgical Oncology, in a paper entitled "Ultrasound examination of the lymphatic drainage area and regional lymph nodes in melanoma patients with in-transit metastasis", Nijhuis and colleagues from the Melanoma Institute Australia (MIA) looked at the utility of ultrasound examination in patients with regionally advanced melanoma. ${ }^{1}$ This group published their retrospective experience on the utility of ultrasound examination in 28 patients with 40 in-transit metastasis, and found, in 15 patients, that ultrasound examination identified additional in-transit lesions that were usually closer to the nodal basin than the existing known disease, compared with clinical examination. They also found no

(C) Society of Surgical Oncology 2020

First Received: 26 October 2020

Accepted: 26 October 2020;

Published Online: 9 November 2020

D. Tyler, MD

e-mail: dstyler@utmb.edu nodal recurrences utilizing ultrasound. Their findings led the group to alter management in 9 of 28 patients, with one false positive ultrasound in this group of patients. The study also describes four false negatives where no lesions were seen with ultrasound in areas where patients ultimately did develop recurrence within 6 months of the last ultrasound examination.

While that group highlights the benefits of ultrasound over clinical examination, one must initially ask what the ultrasound examination really did add over more complete total body staging that most of these patients would already have, such as sentinel lymph node (SLN) biopsy or positron emission tomography/computed tomography (PET/ $\mathrm{CT}$ ). The MIA group has previously reported that ultrasound examination was not accurate at picking up micrometastatic disease in a SLN. ${ }^{2}$ If one looks at the resolution of various imaging techniques such as ultrasound, CT scans, magnetic resonance imaging (MRI) scans, and/or PET/CT scans, it is clear that while the limits of resolution are quite good in the millimeters range, currently no imaging technique can reliably pick up small volumes of malignant disease when the focus is $<2 \mathrm{~mm}$. Despite this limitation, in melanoma patients there is evidence that ultrasound is better than clinical examination in evaluating a nodal basin in patients undergoing surveillance for evidence of recurrence of their melanoma. ${ }^{3}$ Again, the group from MIA found that in patients not thought to be candidates for SLN biopsy due to health issues, or who refused SLN biopsy, ultrasound was able to identify approximately one-third of nodal basin recurrences before they were picked up on clinical examination.

In this patient population of regionally advanced melanoma, one might also wonder what a clinical examination with ultrasound is able to detect or clarify that routine imaging cannot. Once again, the same MIA group has 
looked at their experience with PET/CT in stage III patients with melanoma and concluded that PET/CT for annual surveillance identified metastatic lesions in $13 \%$ of their patients followed under surveillance, but $53 \%$ of patients also had false positive results. ${ }^{4}$ It is certainly acknowledged that there are challenges with CT and PET/CT in identifying small cutaneous and superficial subcutaneous lesions. Lesions that are partially treated may also lose PET avidity and may also be difficult to identify with CT or PET/CT. The potential role of clinical examination and ultrasound over imaging techniques has been previously highlighted by other groups, as Dr Nijhuis points out (with several references in his manuscript). Indeed, there is significant potential in using ultrasound in this patient population to better clarify imaging abnormalities on PET/CT, as well as identifying lesions that were not idenified using these studies. Although several patients in that series underwent CT or PET/CT, there is poor delineation in the manuscript on how ultrasound either correlated with other imaging or helped to resolve potential false positives that were seen with whole-body imaging. In addition, it would have been more helpful to delineate the impact of ultrasound in this patient population if the authors had defined what their operative indications were for surgical excision alone, regional therapy of some form, or systemic therapy in this patient population. Simply finding an additional in-transit lesion in an extremity that already has several lesions does not always alter the clinical decision-making process, especially for cancer programs that may have limited options for treating these types of patients. The ability of ultrasound to better define the burden of disease and help document the location of disease, especially for measuring response to treatment as part of therapeutic interventions or clinical trials, can be particularly helpful, especially for organizations with a robust treatment portfolio.

There are several challenges to gaining more widespread acceptance in ultrasound examination that generally focus on operator dependency for optimal study quality. Some institutions have dedicated imagers who focus only on formal ultrasound examinations in cancer patients. Others have the breast imaging group focus on the axilla and arms, while the abdominal imagers focus on the inguinal basins and lower extremities. The problem is further exacerbated by trying to more widely move ultrasound to the clinic. How do you develop competence in surgeons and trainees who want to perform the examination in their clinics? How do you ensure that high-quality equipment is available? Increasingly, surgical training programs are incorporating ultrasound training and competence certification into the residents' curriculum. Competence in ultrasound should also be incorporated into the curriculum of surgical oncology fellowships. As surgeons become increasingly comfortable in utilizing ultrasound, I suspect they will become the driving force that leads to a further expansion of ultrasound in the clinics. As this occurs, ultrasound will complement the physical examination as an integral component of clinical staging and surveillance. Studies such as those carried out by MIA can help us more clearly understand which patient populations will benefit most from this adjunct imaging modality.

DISCLOSURES Douglas Tyler receives royalties for writing a section on melanoma in-transit disease for UptoDate.

\section{REFERENCES}

1. Nijhuis A, Chung D, Landon K, Uren R, Thompson J, Nieweg O. Ultrasound examination of the lymphatic drainage area and regional lymph nodes in melanoma patients with in-transit metastasis. Ann Surg Oncol. 2020. https://doi.org/10.1245/s10434 $-020-09240-9$

2. Nijhuis A, Dieng M, Khanna N, Lord S, Dalton J, Menzies A, et al. False-positive results and incidental findings with annual CT or PET/CT surveillance in asymptomatic patients with resected stage III melanoma. Ann Surg Oncol. 2019;26:1860-1868.

3. Ipenburg N, Thompson J, Uren R, Chung D, Nieweg O. Focused Ultrasound surveillance of lymph nodes following lymphoscintigraphy without sentinel node biopsy: a useful and safe strategy in elderly or frail melanoma patients. Ann Surg Oncol. 2019;26(9):2855-63

4. Thompson J, Haydu L, Uren R, Andtbacka R, Zager J, Beitsch P, et al. Preoperative ultrasound assessment of regional lymph nodes in melanoma patients does not provide reliable nodal staging: results from a large multicenter study. Ann Surg. 2019. https://doi. org/10.1097/SLA.0000000000003405

Publisher's Note Springer Nature remains neutral with regard to jurisdictional claims in published maps and institutional affiliations. 